\title{
THE INFLUENCE OF GONADECTOMY, ANDROGEN EXPOSURE, OR A GONADAL GRAFT IN THE NEONATAL RAT ON THE VOLUME OF THE SEXUALLY DIMORPHIC NUCLEUS OF THE PREOPTIC AREA ${ }^{1}$
}

\author{
CAROL D. JACOBSON, ${ }^{2}$ VALER J. CSERNUS, ${ }^{3}$ JAMES E. SHRYNE, AND ROGER A. GORSKI ${ }^{4}$ \\ Department of Anatomy and Laboratory of Neuroendocrinology of the Brain Research Institute, UCLA School of Medicine, Los Angeles, \\ California 90024
}

\begin{abstract}
Although the volume of the sexually dimorphic nucleus of the preoptic area (SDN-POA) of the adult rat has been shown to be modified by the hormone environment carly in postnatal life, the present study was performed to clarify several fundamental questions related to this process. This study was designed to evaluate the ability of exogenous testosterone propionate (TP), or a gonadal graft, to influence SDN-POA volume in rats which were gonadectomized as neonates. Orchidectomy on day 1 resulted in an approximately 50\% decrease in adult SDN-POA volume; however, the influence of the testes on the resulting SDN-POA volume was replaced effectively by the administration of $100 \mu \mathrm{g}$ or $1 \mathrm{mg}$ of TP on postnatal day 2 or by a testicular (but not ovarian) graft on the day of castration. In the female, ovariectomy on postnatal day 1 failed to alter SDN-POA volume relative to that of sham-operated females. Exogenous TP, but neither testicular nor ovarian grafts, resulted in a larger SDN-POA volume when observed in the adult female. Thus, the development of the SDN-POA of the neonatal male is significantly influenced by the hormonal activity of the testes at this time period. While the SDN-POA of the neonatal female is potentially responsive to androgen, the role played by the ovaries in the development of the SDN-POA remains unclear. In addition, the different response of the developing male and female SDN-POA to a testicular graft suggests that the hormonal sensitivity of this nucleus may differ in the two sexes.
\end{abstract}

'The fact that the brain undergoes a process of functional sexual differentiation is well documented (see review by Gorski and Jacobson, 1981), but relatively little is known about the basis of this process. Although several subtle morphological differences have been seen in the brains of male and female mammals (see Gorski and Jacobson, 1981), this laboratory has demonstrated the existence of a marked sex difference in the volume of an intensely staining component of the preoptic area of the rat (Gorski et al., 1978). This region has been named the sexually dimorphic nucleus of the preoptic area (SDNPOA) since it has an increased density of neurons com-

\footnotetext{
${ }^{1}$ This work was supported by United States Public Health Service Grant HD-01182 and the Ford, Kroc, and Grant Foundations. We wish to thank Dr. Arthur Arnold for the use of a mini-computer system for the evaluation of nuclear volume, Erna Freiberg for preparing excellent histological specimens, and Nicole Lotwin for typing the manuscript.

${ }^{2}$ Predoctoral trainee of the Mental Health Training Program, United States Public Health Service Grant MH-15345, when the research was conducted. Present address: Department of Veterinary Anatomy, School of Veterinary Medicine, Iowa State University, Ames, IA 50011.

${ }^{3}$ Fellow of the Ford Foundation. Present address: Department of Anatomy, University Medical School, Pecs, Hungary.

${ }^{4}$ To whom correspondence should be addressed at Department of Anatomy, UCLA School of Medicine, Los Angeles, CA 90024.
}

pared to that of its surround as seen in Nissl-stained sections (Gorski et al., 1980).

Furthermore, this sexual dimorphism in volume gradually develops during the first 10 days of postnatal life (Jacobson et al., 1980), which is a time period critical for the functional sexual differentiation of the brain. The volume of the SDN-POA appears to be independent of the steroidal environment in the adult but can be influenced significantly by the steroid hormone milieu during early postnatal life (Gorski et al., 1978). Castration of the 1-day-old male pup will reduce the volume of the SDNPOA significantly as seen in the adult. Conversely, administration of $1 \mathrm{mg}$ of testosterone propionate (TP) to the 4-day-old intact female pup will increase the volume of the SDN-POA significantly as seen in the adult. In neither case, however, was the volume of the SDN-POA totally determined by the postnatal steroid environment, i.e., the volume of the neonatally castrated male's SDNPOA is still larger than that of the normal female. In order to investigate further the role of gonadal steroids during the early postnatal period on SDN-POA development, the present study was conducted. Attempts were made to reverse the effect of orchidectomy by administering exogenous TP or by implanting a gonadal graft within 2 days of birth. In addition, we hoped to evaluate 
TABLE I

Sequence of experimental treatment

\begin{tabular}{clccc}
\hline Group $^{\prime \prime}$ & \multicolumn{1}{c}{ Day 1 } & Day 2 & Day 45 & Day 60 \\
\hline 1 & Intact & & Gx & Gx \\
2 & Sham Gx & Oil & Graft removed & Sacrifice \\
3 & Gx + ovarian graft & & Graft removed & Sacrifice \\
4 & Gx + testicular graft & Oil & Sham surgery & Sacrifice \\
5 & $\mathrm{Gx}$ & $100 \mu$ of $\mathrm{TP}$ & Sham surgery & Sacrifice \\
6 & $\mathrm{Gx}$ & $1 \mathrm{mg}$ of TP & Sham surgery & Sacrifice \\
7 & $\mathrm{Gx}$ & &
\end{tabular}

" Hach group was subdivided further by sex.

the importance of the ovary during the early postnatal period for the establishment of the female type SDNPOA and to determine if exogenous steroids or a gonadal graft given to the neonatally gonadectomized female would increase the adult volume of the SDN-POA.

\section{Materials and Methods}

Pregnant Sprague-Dawley females were obtained from Simonsen Laboratories (Gilroy, CA) and maintained on a 14:10 light:dark cycle with food and water available ad libitum. The day of birth was defined as day 1 of postnatal life. On this day, both male and female pups were assigned to one of seven groups (Table I). The groups consisted of untreated control animals or pups which were gonadectomized $(\mathrm{Gx})$ or sham operated under cryoanesthesia on that day. Additionally, either a single testis or ovary from their littermates was implanted subcutaneously in the back of the neck in some of the $\mathrm{Gx}$ animals. On day $2, \mathrm{Gx}$ pups which did not receive gonadal grafts were given a single injection of either oil vehicle or $100 \mu \mathrm{g}$ or $1 \mathrm{mg}$ of TP. Sham-operated pups also received an injection of oil. On day 45, under ether anesthesia, gonadal grafts were removed; all other rats were subjected to sham surgery (a skin incision). Gonadal grafts were fixed in Bouin's solution, embedded in paraffin, scrially sectioned at $8 \mu \mathrm{m}$, and stained with hematoxylin and eosin. Following histological observations, all animals which did not have viable testicular or ovarian grafts were dropped from the study. Hormonal sensitivity for the induction of lordosis behavior was studied in all groups of animals. ${ }^{5}$

One to 2 months following behavioral testing, the body weights of a randomly selected subset of these animals were obtained. These animals then were sacrificed under ether and perfused intracardiacally with saline followed by $10 \%$ formalin. After removal of the brain from the calvaria and trimming, brain weights were obtained. A total of 58 brains were analyzed in the present study.

Following postfixation in $10 \%$ formalin, brains were frozen sectioned at $60 \mu \mathrm{m}$ using the deGroot plane (deGroot, 1959) and stained with thionin. After these histological procedures, the slides were coded hy animal

\footnotetext{
${ }^{5}$ The behavioral responsiveness to estradiol benzoate (EB) was similar (and low) in all groups. Adult treatment of the neonatally $\mathrm{Gx}$ female or male with EB plus progesterone markedly facilitated lordosis responding. However, early treatment of similarly $\mathrm{Gx}$ animals with testosterone propionate, but not gonadal grafts (either testicular or ovarian), suppressed lordosis responding following priming of the adult with EB plus progesterone (J. Csernus and R. A. Gorski, unpublished observations).
}

according to a random number generator so that all further analyses were performed without knowledge of treatment. However, male and female brains were analyzed separately in this study. The volume of the SDNPOA for each rat was determined as described previously (Gorski et al., 1978; Jacobson et al., 1980). Once the average drawing for each section of the SDN-POA was obtained, nuclear area was determined by using a Summagraphic bit pad attached to a Sol mini-computer system. The volume of the SDN-POA was obtained by summing the area of the individual nuclear tracings for each animal after taking into account section thickness and the magnification of the microprojector utilized in obtaining the drawings. Statistical analyses of all data were performed with computer assistance and involved one-way analysis of variance (ANOVA; Dixon and Brown, 1979) and the Duncan's Multiple Range test (Bruning and Kintz, 1977). For our analyses, $p$ values of 0.05 or less were accepted as significant.

\section{Results}

Since brains from animals of known sex were evaluated in this study, all analyses conducted were limited to determining the effects of the various treatments on the volume of the SDN-POA in each sex. Nevertheless, as can be seen from the data presented below, there is an obvious sex difference in SDN-POA volume for rats which received the same treatment.

Analysis of the SDN-POA volume in the male. Although the mean body weights of male rats at sacrifice were significantly $(p<0.0001)$ less in the neonatally gonadectomized animals which received either oil or gonadal grafts as compared to those of the other groups, brain weights obtained following perfusion did not differ in any of the groups (Table II). As can be seen in Figure 1 , in confirmation of our earlier report (Gorski et al., 1978), the volume of the SDN-POA of the adult male rat was influenced by gonadal steroids during early postnatal life. One-way ANOVA of these data yielded a highly significant main effect of treatment $(p<0.0001)$. Further analysis by Duncan's Multiple Range test revealed that the volume of the SDN-POA in the control and shamoperated groups is significantly greater than that of the $\mathrm{Gx}+$ oil or Gx + ovarian graft groups. Also, $100 \mu \mathrm{g}$ or 1 $\mathrm{mg}$ of TP administered on day 2 of postnatal life or a testicular graft implanted on day 1 completely reversed the effects of castration since the volume of the SDNPOA in these adult rats is equal to that of the shamoperated group. However, the presence of an ovarian graft did not significantly affect the resulting adult vol- 
TABLE II

The influence of neonatal gonadectomy, testosterone propionate administration, or implantation of a gonadal graft on the resulting body weight and brain weight of adult animals at the time of sacrifice

\begin{tabular}{|c|c|c|c|c|c|c|}
\hline \multirow[b]{2}{*}{ Group } & \multicolumn{3}{|c|}{ Males } & \multicolumn{3}{|c|}{ Females } \\
\hline & No. & $\begin{array}{c}\text { Body } \\
\text { Weight }\end{array}$ & $\begin{array}{c}\text { Brain } \\
\text { Weight }\end{array}$ & No. & $\begin{array}{c}\text { Body } \\
\text { Weight }\end{array}$ & $\begin{array}{c}\text { Brain } \\
\text { Weight }\end{array}$ \\
\hline & \multicolumn{4}{|c|}{$g m$} & \multicolumn{2}{|c|}{$g m$} \\
\hline Intact & 3 & $440.0 \pm 9.2$ & $1.92 \pm 0.02$ & 4 & $337.5 \pm 15.8$ & $1.96 \pm 0.06$ \\
\hline Sham Gx + oil & 4 & $452.3 \pm 22.7$ & $1.91 \pm 0.04$ & 6 & $344.8 \pm 11.3$ & $1.93 \pm 0.05$ \\
\hline $\mathrm{Gx}+$ ovarian graft & 4 & $346.0 \pm 18.5^{a}$ & $1.95 \pm 0.02$ & 6 & $287.8 \pm 17.6^{b}$ & $1.79 \pm 0.05$ \\
\hline Gx + testicular graft & 3 & $366.7 \pm 3.3^{a}$ & $1.93 \pm 0.02$ & 4 & $336.5 \pm 14.0$ & $1.93 \pm 0.03$ \\
\hline $\mathrm{Gx}+$ oil & 4 & $370.5 \pm 7.7^{a}$ & $1.90 \pm 0.05$ & 4 & $371.0 \pm 10.9$ & $1.95 \pm 0.06$ \\
\hline $\mathrm{Gx}+\mathrm{I} 00 \mu \mathrm{g}$ of TP & 4 & $440.0 \pm 10.2$ & $1.98 \pm 0.03$ & 5 & $379.6 \pm 15.5$ & $1.93 \pm 0.02$ \\
\hline $\mathrm{Gx}+1 \mathrm{mg}$ of TP & 3 & $482.0 \pm 36.4$ & $2.05 \pm 0.08$ & 4 & $381.0 \pm 7.0$ & $1.95 \pm 0.02$ \\
\hline
\end{tabular}

"Significantly less than sham $\mathrm{Gx}+$ oil.

"Significantly less than all other groups.

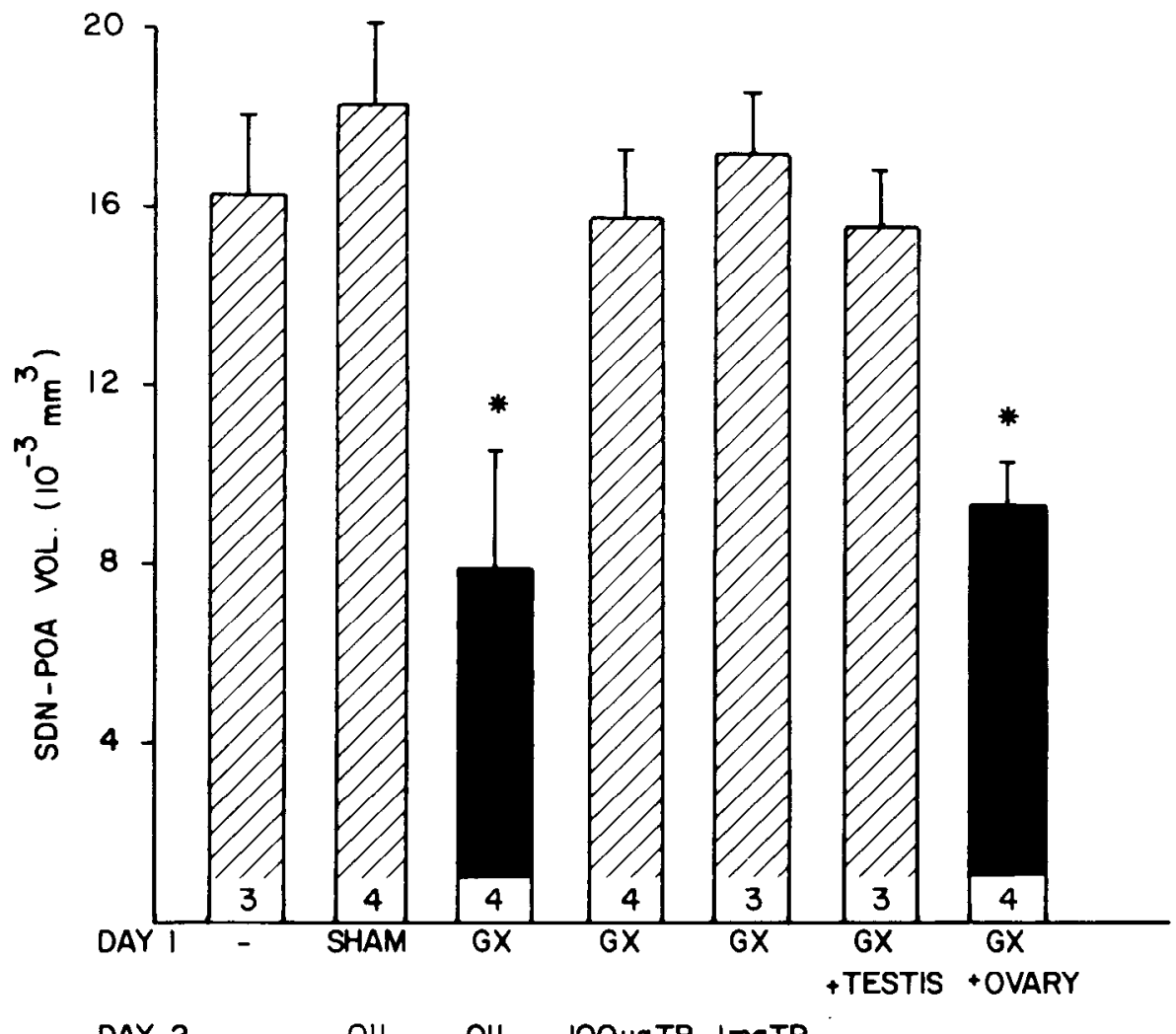

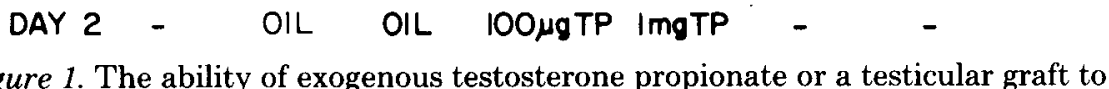
Figure 1. The ability of exogenous testosterone propionate or a testicular graft to
reverse the influence of gonadectomy of the male rat on day 1 of postnatal life on the volume of the sexually dimorphic nucleus of the preoptic area when measured in adulthood. The asterisks indicate values which are significantly less than all other groups represented by the shaded bars. The number at the base of each bar represents the number of brains analyzed.

ume of the SDN-POA of the neonatally orchidectomized rat.

Analysis of the SDN-POA volume in the female. Although the mean body weight of neonatally gonadectomized females given a subcutaneous ovarian graft was significantly $(p<0.0001)$ less than that of any other group, brain weights obtained following perfusion did not differ among any of these groups (Table II). Figure 2 illustrates that the volume of the SDN-POA of the adult female, as in the male, is influenced by gonadal steroids during the first days of postnatal life. One-way ANOVA revealed a highly significant main effect of treatment ( $p$ $<0.0001$ ). Further analysis by Duncan's Multiple Range test revealed that the volume of the SDN-POA of the neonatally ovariectomized + oil-treated rats is reduced significantly compared to that of the group of intact females. However, the volume of the SDN-POA of the $\mathrm{Gx}+$ oil group does not differ from that of the shamoperated or $\mathrm{Gx}+$ gonadal grafted rats. Also, the administration of either $100 \mu \mathrm{g}$ or $1 \mathrm{mg}$ of TP significantly 


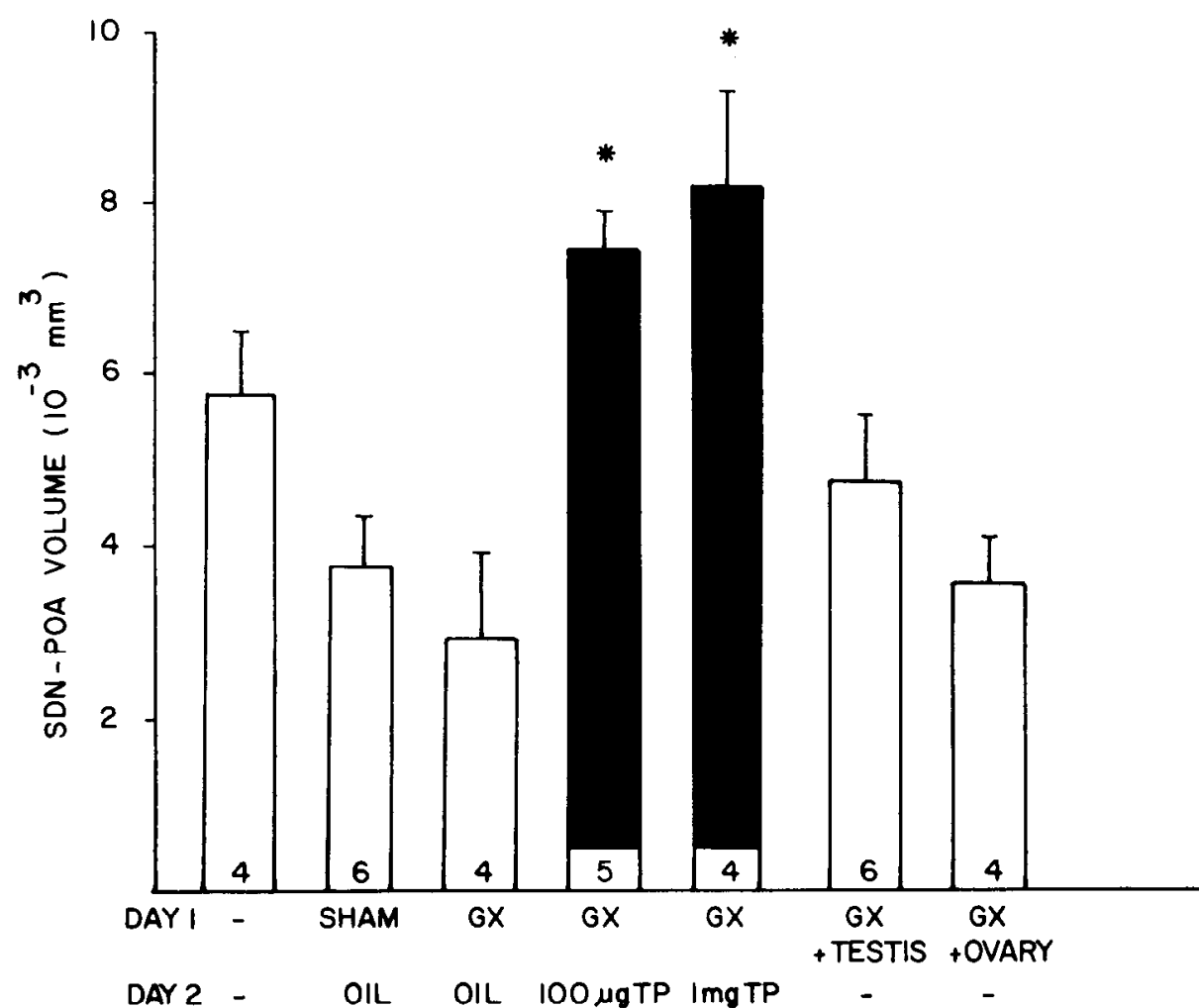

Figure 2. The ability of exogenous testosterone propionate to potentiate the volume of the sexually dimorphic nucleus of the preoptic area of the neonatally ovariectomized female when measured in adulthood. The asterisks indicate values which are significantly greater than the group of sham-operated (SHAM) females. The number at the base of each bar represents the number of brains analyzed.

increases the volume of the SDN-POA compared to that of the $\mathrm{Gx}+$ oil group.

\section{Discussion}

The results of the present study confirm and extend previous data and emphasize the importance of the steroidal environment in the rat during the first days of postnatal life, a time period important for the functional sexual differentiation of the brain and the morphological differentiation of the SDN-POA. Previously, gonadectomy of the 1-day-old male was shown to inhibit the development of the SDN-POA in terms of its volume as seen in the normal male adult (Gorski et al., 1978). The present results demonstrate that a single injection of either $100 \mu \mathrm{g}$ or $1 \mathrm{mg}$ of TP given to the neonatal male 1 day after gonadectomy can effectively replace the endogenous hormonal milieu which is necessary during this critical period for the maturation of this nucleus.

Although mitosis of the neuroblasts destined to form the SDN-POA is apparently prolonged relative to that of neuroblasts destined for the surrounding medial preoptic area and although there may be steroid influences on neurogenesis (Jacobson and Gorski, 1981), our unpublished data suggest that neurogenesis of the SDN-POA is complete by the time of birth. The present data, therefore, suggest that approximately 50\% (see Fig. 1) of the volume of the SDN-POA is completely dependent on the early postnatal hormonal environment in spite of the postmitotic state of the neurons of the SDN-POA. It is interesting to note that the administration of exogenous TP to the intact male pup on postnatal day 2 does not increase the volume of the SDN-POA significantly as seen in the adult (Jacobson, 1980). Thus, it is possible that there is a maximum volume that can be obtained by the SDN-POA under hormonal influence postnatally.

Previous data also have shown that a single injection of $1 \mathrm{mg}$ of TP given on day 4 of postnatal life to the intact female will result in a significantly larger adult SDNPOA volume when compared to the volume of the nucleus of the vehicle-injected female (Gorski et al., 1978). The present results confirm this in the ovariectomized rat given either $100 \mu \mathrm{g}$ or $1 \mathrm{mg}$ of TP on day 2 . However, even though the volume of the SDN-POA is increased by the injection of TP, the volume of the nucleus of the androgen-injected female does not approach the volume of the male nucleus. Since the ability to sex-reverse the volume of the SDN-POA may be a function of the time of exposure to the hormone (i.e., the earlier the TP is administered, the more potent its effects would be), a study is being conducted presently in order to determine if the prenatal hormone environment can influence the ultimate volume of the SDN-POA as seen in the adult. This is considered possible since it has been shown that the intrauterine position of the female mouse (Gandelman et al., 1977) or rat (Clemens et al., 1978) fetus will affect its later reproductive performance. The amniotic fluid of the female mouse fetus which is located between two males in utero has been shown to have significantly 
higher testosterone titers than that of the female which is located between two females (vom Saal and Bronson, 1980a). The effects of the locally high testosterone levels can be seen at birth since the anogenital distance is greater in these animals as compared to that of the control females (Clemens et al., 1978; vom Saal and Bronson, 1980a). Furthermore, as adults, these animals have been reported to show a greater incidence of irregular vaginal cycles (vom Saal and Bronson, 1980b). Although the volume of the SDN-POA is clearly influenced by steroids during early postnatal life, the precise role of the prenatal hormone environment remains to be clarified.

The response of the developing SDN-POA of the neonatally $\mathrm{Gx}$ animals which received a gonadal graft differed in the male and female. The volume of the SDNPOA of the neonatally $\mathrm{Gx}$ male which received a testicular graft is equal to that of the sham-operated male and, as well, to that of the groups of neonatally $\mathrm{Gx}$ males which received either $100 \mu \mathrm{g}$ or $1 \mathrm{mg}$ of TP. In the female, administration of either $100 \mu \mathrm{g}$ or $1 \mathrm{mg}$ of TP more than doubles the volume of the SDN-POA when compared to that of the oil-treated neonatally Gx female. However, a testicular graft implanted in the neonatally ovariectomized rat does not result in a significantly greater SDNPOA volume. The differing response of the developing male and female SDN-POA to the presence of a testicular graft suggests a differential sensitivity to the steroidal environment since, in both sexes, exogenous androgen (presumably at supraphysiological levels) is able to affect nuclear volume. Further studies utilizing various doses of TP are required to verify this conclusion. Recently, it has been shown that steroid receptors are present in the prenatal and neonatal male and female rat brain (MacLusky et al., 1979; Vito and Fox, 1979). Furthermore, it appears that there is a greater occupancy of nuclear estrogen receptors in the developing male brain than in that of the female (Lieberburg et al., 1979). Therefore, subsequent studies also are required to determine if the presumed differential sensitivity of the developing SDN-POA of the male and female rat is due to a quantitative difference in steroid receptors or in their occupancy.

As stated above, although ovariectomy of the 1-dayold rat significantly reduced SDN-POA volume as compared to that of the intact and untreated control, ovariectomy had no effect on SDN-POA volume when compared with that of the sham-operated female. Since it is possible that sham surgery may disrupt the functional activity or the neural control of the ovary or the metabolism of ovarian hormones, the present data do not deny conclusively the importance of the ovaries during the early postnatal period for the development of the SDNPOA in terms of its final volume. In fact, several reports suggest that the ovaries are important for some parameters of brain maturation, including myelinization (Casper et al., 1967), synaptogenesis (Matsumoto and Arai, 1976), and a marked proliferation of neuropil (Naftolin and Brawer, 1978). Furthermore, Toran-Allerand (1976, 1980) has demonstrated very elegantly the importance of estradiol for the growth of neurite processes from explants of newborn mouse hypothalamus. In addition, the neurite outgrowths appear to emanate from steroid responsive neurons (Toran-Allerand et al., 1980).

Finally, Döhler (1978) has suggested that exposure to ovarian hormones may be a prerequisite for the normal differentiation of the female brain. Recently, we have shown that the volume of the SDN-POA of females exposed to the antiestrogen (tamoxifen) during the early postnatal period is significantly smaller than that of the vehicle-injected control female (Döhler et al., 1981). Thus, testicular and ovarian hormones may be necessary for the differentiation of the normal male and female SDN-POA, respectively. However, further studies are necessary to define the origin of estrogen during the postnatal period and its precise role in the processes of development, differentiation, and maturation of the SDN-POA.

\section{References}

Bruning, J. L., and B. L. Kintz (1977) Computational Handbook of Statistics, Scott, Foresman and Co., Glenview, IL.

Casper, R., A. Vernadakis, and P. S. Timaras (1967) Influence of estradiol and cortisol on lipids and cerebrosides in the developing brain and spinal cord of the rat. Brain Res. 5: 524-526.

Clemens, L. G., B. A. Gladue, and L. P. Coniglio (1978) Prenatal endogenous androgenic influences on masculine sexual behavior and genital morphology in male and female rats. Horm. Res, 10: 40-53.

deGroot, J. (1959) The rat forebrain in stereotaxic coordinates, Verh. K. Ned. Akad. Wet. Afd. Natuurk. D. 'Iweede Reeks 52: $1-40$.

Dixon, W. J., and M. B. Brown (1979) Biomedical Computer Program, P. Series, University of California Press, Berkeley.

Döhler, K. D. (1978) Is female sexual differentiation hormonemediated? Trends Neurosci. 1: 138-140.

Döhler, K. D., S. S. Srivastava, R. A. Gorski, and J. Shryne (1981) Postnatal tamoxifen treatment interferes with differentiation of the sexually dimorphic nucleus of the preoptic area in both male and female rats. Endocrinology (Suppl.) 108: 204.

Gandelman, R., F. S. vom Saal, and J. M. Reinisch (1977) Contiguity to male fetuses affects morphology and behaviour of female mice. Nature 266: 722-724.

Gorski, R. A., and C. D. Jacobson (1981) Sexual differentiation of the brain. In Clinics in Andrology: Pediatric Andrology, S. J. Kagen and E. S. E. Hafez, eds., Vol. III, pp. 109-134, Nijhoff, The Netherlands.

Gorski, R. A., J. H. Gordon, J. E. Shryne, and A. M. Southam (1978) Evidence for a morphological sex difference within the medial preoptic area of the rat brain. Brain Res. 148: 333346.

Gorski, R. A., R. E. Harlan, C. D. Jacobson, J. E. Shryne, and A. M. Southam (1980) Evidence for the existence of a sexually dimorphic nucleus in the preoptic area of the rat. J. Comp. Neurol. 193: 529-539.

Jacobson, C. D. (1980) The characterization, ontogeny and influence of androgen on the sexually dimorphic nucleus of the preoptic area. Doctoral dissertation, University of California, Los Angeles.

Jacobson, C. D., and R. A. Gorski (1981) Neurogenesis of the sexually dimorphic nucleus of the preoptic area of the rat. J. Comp. Neurol. 196: 519-529.

Jacobson, C. D., J. E. Shryne, F. Shapiro, and R. A. Gorski (1980) Ontogeny of the sexually dimorphic nucleus of the preoptic area. J. Comp. Neurol. 193: 541-548.

Lieberburg, I., L. C. Krey, and B. S. McEwen (1979) Sex 
differences in serum testosterone and in exchangeable brain cell nuclear estradiol during the neonatal period in rats. Brain Res. 178: 207-212.

MacLusky, N. J., I. Lieberburg, and B. S. McEwen (1979) The development of estrogen receptor systems in the rat brain: Perinatal development. Brain Res. 178: 129-142.

Matsumoto, A., and Y. Arai (1976) Effect of estrogen on early postnatal development of synaptic formation in the hypothalamic arcuate nucleus of female rats. Neurosci. Lett. 2: 7982.

Naftolin, F., and J. R. Brawer (1978) The effect of estrogens on hypothalamic structure and function. Am. J. Obstet. Gynecol. 132: 758-765.

Toran-Allerand, C. D. (1976) Sex steroids and the development of the newborn mouse hypothalamus and preoptic area in vitro: Implications for sexual differentiation. Brain Res. 106: 407-412.
Toran-Allerand, C. D. (1980) Sex steroids and the development of the newborn mouse hypothalamus and preoptic area in vitro: II. Morphological correlates and hormonal specificity. Brain Res. 189: 413-427.

Toran-Allerand, C. D., J. L. Gerlach, and B. S. McEwen (1980) Autoradiographic localization of $3 \mathrm{H}$-estradiol related to steroid responsiveness in cultures of the newborn mouse hypothalamus and preoptic area. Brain Res. 184: 517-522.

Vito, C. C., and T. O. Fox (1979) Embryonic rodent brain contains estrogen receptors. Science 204: 517-519.

vom Saal, F. S., and F. H. Bronson (1980a) Sexual characteristics of adult female mice correlate with their blood testosterone levels during prenatal development. Science 208: 597599.

vom Saal, F. S., and F. H. Bronson (1980b) Variation in length of the estrous cycle in mice due to former intrauterine proximity to male fetuses. Biol. Reprod. 22: 777-780. 